\title{
P 14 IT'S GETTING HARDER TO BREATH: NON-INVASIVE VENTILATION (NIV) IN ADVANCED CHRONIC OBSTRUCTIVE PULMONARY DISEASE (COPD)
}

N. Khan', D. Munday' ${ }^{1}$ University of Warwick

10.1136/bmjspcare-2012-000264.21

Introduction Domiciliary non-invasive positive-pressure ventilation (NIPPV) is increasingly employed to provide respiratory support in chronic respiratory failure (CRF) due to COPD. Whilst there are associated positive financial implications in a particular sub-section of patients due to reduction in hospital admission stays, significant debate remains around its long-term usefulness in COPD patients.

Aims and Methods 1. Has health-related quality of life (HROL) been assessed in severe COPD patients undergoing domiciliary NIPPV? 2. If so, what is the impact of domiciliary NIPPV on the HRQL of severe COPD patients? 3. What are the attitudes of COPD patients towards receiving NIV to palliate breathlessness during end-stage disease? Literature review and narrative analysis.

Results A small number of studies have been identified that have assessed HRQL impacts of domiciliary NIPPV as a secondary outcome. However, there was wide methodological heterogeneity in NIPPV use and the HRQL tools used. There is a suggestion that severe COPD patients requiring NIPPV have a worse psychological status than non-COPD patients requiring NIPPV. NIPPV may help to reduce sleep disturbance and improve components of HRQL scores, however evidence is not conclusive. No studies were found that attempted to explore the attitudes of COPD patients towards receiving NIV to palliative breathlessness in terminal disease. Conclusion: HRQL has been assessed in severe COPD patients as part of studies that aim to assess health-related outcomes of undergoing domiciliary NIPPV. However, evidence regarding the role of domiciliary NIV in improving HROL in end-stage COPD is conflicting due to a lack of adequate studies designed to answer this question primarily and methodological heterogeneity in existing studies. No qualitative research has been carried out to address this question. There is a need for qualitative studies that examine COPD patients attitudes towards NIV as a palliative measure, as it may be of relevance for patients in the decision-making process as part of advance care planning in COPD. 